\title{
EDITORIAL
}

\section{Safety of cardiovascular magnetic resonance in patients with cardiovascular implants and devices}

\author{
S K Prasad, D J Pennell
}

Cardiovascular magnetic resonance is very safe, but occasional serious complications may occur in patients with some implants or devices. Pacemakers currently present the largest problem, but new research shows that the perceived dangers have been overestimated and in particular new lead solutions are emerging. Common implants that may be scanned safely include sternal and epicardial wires, prosthetic valves, and stents.

Heart 2004;90:1241-1244. doi: 10.1136/hrt.2003.021154

while some metals and all non-metals are diamagnetic, indicating that they are slightly repelled by a magnetic field. Clinical issues of safety of CMR relate only to devices with ferromagnetic components. It is important, however, to distinguish between iron and high grade steel. Iron is ferromagnetic whereas the steel used in human implants is usually not, because of impurities added to the iron to give it strength and prevent rusting.

\section{Magnetic field interactions}

Any ferromagnetic object placed in a magnetic field will be subject to forces of deflection, attraction, and torque. This has the potential to lead to injury in two ways. The first potential for injury is from projectiles. Objects made with low grade steel which retains ferromagnetic properties, such as scissors, oxygen cylinders, and wheelchairs, can be attracted into the magnet. These and other metallic objects are therefore routinely banned from MR suites. Specially designed wheelchairs, trolleys, and anaesthetic equipment made of aluminium are available and must be unequivocally labelled as MR safe, and not released from the MR suite. As a corollary to this, resuscitation of patients suffering cardiac arrest in the MR suite must begin with evacuation of the patient from the MR scanner room.

The second potential for injury is with implanted devices, because of the possibility of movement. Translational attraction is measured by the deflection angle test. ${ }^{2}$ Factors affecting it are mainly the strength of the magnetic field, the degree of ferromagnetism of the object, its mass and location, and the presence of retentive tissues such as fibrosis, skin, sutures, or bone. At 1.5T, CMR can be performed safely if the metallic object is non-ferromagnetic or is weakly ferromagnetic, whereby it is only minimally attracted by the magnetic field in relation to its in vivo application. That is to say that the attractive force is insufficient to move the implant in situ or affect its intended function. Ex vivo evaluation of devices by manufacturers and safety regulatory bodies provides information on this. An example of a safe implant would be a hip prosthesis. One example where there may be problems is the neurovascular clip. These are placed on delicate neural vessels-for example, after subarachnoid haemorrhage - and cases of dislodgement have occurred. If CMR is required in such patients, it is usually possible, but advice should be taken from expert centres

Abbreviations: CMR, cardiovascular magnetic resonance; ICD, implantable cardioverter-defibrillator; $\mathrm{RF}$, radiofrequency; T, Tesla 
with full details of the manufacturer and type of clip implanted.

\section{Induced electrical currents}

CMR of patients with devices containing conducting materials exposes these materials to RF and pulsed gradient magnetic fields which have the potential to cause harm by inducing electrical currents. RF pulses can induce heating if there is sufficient coupling to the RF wavelength. This includes pacing wires, guide wires, and indwelling catheters with metallic components such as thermodilution catheters. ${ }^{3}$ Pacemaker leads are a particular issue because of their chronic indwelling use and their length, which at high field strengths begins to approach the wavelength of the RF. This may allow significant coupling and therefore heating. The dimensions, orientation, shape, and location of the metallic implant all play a role. ${ }^{4}$ Finally, exposure to the electromagnetic field used by CMR scanners may inhibit or modify the operational or functional settings of an electronic implant.

\section{SAFETY OF CARDIOVASCULAR DEVICES Patient screening before CMR}

Proper screening of patients before CMR is essential, and this should be in the form of a questionnaire, ${ }^{5}$ which is completed and signed by the patient, and cross signed by the CMR staff.

\section{ECG leads}

Standard metallic ECG tabs may induce burns during CMR, and should not therefore be used. Carbon based ECG electrodes and leads with a higher impedance are available which are safe for CMR and do not produce artefact.

\section{Sternal and epicardial pacing wires}

Scanning patients with sternal wires is safe. ${ }^{67}$ In patients with retained epicardial pacing wires following cardiac surgery, there are no reports of adverse events or arrhythmias during CMR up to $1.5 \mathrm{~T}$ with conventional pulse sequences. ${ }^{8}$ There is a small artefact but this is localised and rarely interferes with image analysis.

\section{Heart valve prostheses and annuloplasty rings}

At $1.5 \mathrm{~T}$, all heart valve prostheses and annuloplasty rings are CMR compatible. ${ }^{9}$ Those tested at $3 \mathrm{~T}$ so far are also compatible. ${ }^{2}$ Magnetic field interactions are either nil or very small and are minimal compared to the force exerted by the beating heart. Induced currents and RF heating do not appear to be problematic. ${ }^{10}$ No documented adverse patient incidents have been reported. Previous concerns about the Starr-Edwards Pre-6000 prosthesis were unfounded. Prosthetic valves cause localised artefact but this rarely affects image interpretation.

\section{Coronary stents}

CMR could in theory lead to heating or movement of coronary stents. Sophisticated in vitro trials show that the heating effect is tiny, and would have no clinical effects. ${ }^{11}$ Dislodgement is also a possibility that could, in principle, increase exposure of metallic coronary stent material to platelets which might trigger stent thrombosis. However, the majority of available coronary stents are made from non- or weakly ferromagnetic materials (316L stainless steel, nitinol, or titanium), and in vitro trials show very minimal magnetic attraction. ${ }^{12}$ In the Mayo series of patients who underwent MR at $1.5 \mathrm{~T}$ for severe co-morbidity less than eight weeks after standard coronary stent placement, no case of thrombosis was identified and there was no increased risk of mortality over a 30 day period post-deployment. ${ }^{13}$ In a study of patients at day 3 post-coronary stent deployment, there was no increase in adverse events compared to control groups after seven months follow up. ${ }^{14}$ These clinical studies support animal work showing that coronary stent migration is absent or minimal. ${ }^{15}$

The development of new mixed alloy coronary stents incorporating cobalt and other metals will need evaluation for CMR compatibility, but as the mass of the stent is small, it is unlikely that results will be different. Therefore, for correctly deployed coronary stents, there is a very low likelihood of movement or heating and it is safe to perform CMR immediately after placement. However, the product insert for many of these coronary stents indicates a period of 6-8 weeks after placement before CMR. We have found nothing in the published literature to support this apparently arbitrary assertion and there are no reports of attributable adverse events associated with early CMR. Established practice worldwide is for CMR to be performed at any time after implantation, and all the available evidence supports this approach. A small artefact may be seen at the site of a coronary stent, which is sometimes useful for localisation.

\section{Other vascular stents, and coils and filters}

Non-coronary intravascular stents (such as those used in the aorta), as well as coils and filters can be imaged at any time after implantation if they are non-ferromagnetic. ${ }^{3} 16$ For stents which have some magnetic attraction, it has been stated that it is prudent to delay CMR for approximately six weeks, ${ }^{3}$ although there is no evidence to support this assertion, and early CMR has been reported without adverse effect. $^{17}$

\section{Occlusion devices}

Metallic occlusion devices are used for closure of shunts. Provided the correct sized device has been deployed, the retentive forces due to the shape of the device and later from tissue growth outweigh those from the applied magnetic field. When the device is made of non-ferromagnetic alloy (for example, MP35N), patients may be scanned immediately after implantation. For devices made of weakly ferromagnetic steel, a delay of six weeks has been recommended but evidence in this area is very limited. ${ }^{18}$

\section{Cardiovascular catheters}

Ex vivo evaluation of a number of different catheters has been carried out. Catheters with any form of metallic component including internal or external conductive wires present a contraindication to $\mathrm{CMR}^{7}$ In general those made out of polyurethane and polyvinyl chlorides are safe. Certain thermodilution pulmonary flotation catheters include a conductive wire which may malfunction and cause injury through excessive heating. ${ }^{5}$

\section{Guidewires}

Conventional guidewires are made from stainless steel or nitinol. The RF may induce excessive heating and consequent damage. For the growing field of interventional CMR, specially designed guidewires with RF decoupling are used which restricts the heating. ${ }^{19}$ Care should also be exercised with the choice of sequences used.

\section{Intra-aortic balloon pumps and left ventricular assist} devices

Due to their high metallic content, these devices with their associated equipment are not considered suitable for CMR, although there is little evidence in the literature. Problems include mechanical malfunction, heating, and the risk of tissue damage.

\section{Pacemakers}

The issue of CMR in patients with pacemakers is not straightforward and is currently in a stage of re-evaluation and change. The simplest summary edict is that in 2004, 
"pacemakers are a strong relative contraindication to CMR". However, this statement merits further expansion in order to explain the absence of "absolute contraindication", which many would consider appropriate.

The reasons for the contraindication of CMR in patients with pacemakers are at least fivefold. First, the magnetic field may cause movement of the pacing box. This is not particularly problematic, because experience has shown that the pacemaker pouch has sufficient strength to hold the pacemaker in position, but if necessary, superficial strapping can be used. Second, RF induced heating of the pacing leads might cause injury to the myocardium or vessels. This is known to be significant and in the experimental setting, and lead temperatures of more than $60^{\circ} \mathrm{C}$ have been recorded. ${ }^{20}$ While this is ameliorated in vivo by the constant flow of blood over the lead, this clearly is of concern. Third, the RF and gradient magnetic fields may induce interference with the electronic circuits of the pacemaker, ${ }^{21}$ or induce currents in the pacemaker lead which might be interpreted by the pacemaker as myocardial electrical activity leading to inhibited or very rapid pacing. Fourth, at least in older pacemakers, both temporary and permanent alteration in pacemaker settings have been described. ${ }^{22}$ Finally, the most important evidence for the contraindication of CMR in pacemaker patients is the documented mortality that has occurred. ${ }^{23-25}$ It is thought that this was related to pacing inhibition, malfunction of the magnetic reed switch, or induction of rapid pacing rates that are incompatible with sustaining cardiac output as a consequence of induced electrical currents. ${ }^{726}$

However, having noted all of the above problems and the documented mortality, there are now a growing number of more up-to-date reports of CMR being performed safely in pacemaker patients in field strengths from $0.35-1.5 \mathrm{~T},{ }^{27-30}$ with 54 patients in the largest and most recent trial at 1.5T. ${ }^{31}$ How can this be explained? One key issue is that the mortality reports suggest that the heart was not the organ under examination by MR. Although not explicitly stated, it is highly likely that this means that no ECG was being monitored during the MR, and that no measures were taken to reduce the risk of the MR scan. In the modern environment, CMR is always performed with continuous ECG monitoring, which would readily identify any arrhythmia. In addition, blood pressure and oxygen saturation monitoring are normally available in CMR centres. Under these circumstances, it is inconceivable that a patient could die unnoticed as a result of cardiovascular collapse from pacemaker malfunction. Secondly, we and others have shown that in non-pacemaker dependent patients who have implanted pacemakers, the pacemaker can be turned off for the duration of the scan and the lead set to bipolar mode. ${ }^{29} 30$ No deaths and no significant complications have resulted from CMR scans being performed in this manner at low field, gradient, and RF sequence settings.

Thus returning to the issue of whether the presence of an implanted pacemaker is an absolute contraindication to CMR, we are forced to conclude that since safe scanning has been performed in several centres, that it can only be the case that CMR is a strong relative contraindication. However, it is important that there is no mistaking our position on this issue. The fact that it is even possible for pacemaker patients to die while undergoing MR explicitly means that such a procedure should not be undertaken outside of expert CMR centres with full pacing facilities, in circumstances where the balance of benefit unequivocally outweighs the risk, and after full informed written consent has been obtained from the patient. Our experience is that few patients fall into this category, although many patients with pacemakers are undoubtedly disadvantaged by having CMR declined. In the event of inadvertent exposure of a pacemaker patient to MR, it is important to check the pacing function as soon as possible.

\section{Implantable cardioverter-defibrillators (ICDs)}

ICDs are subject to similar risks of pacemakers but notably with greater translational attraction. ${ }^{32}$ There is one report of the safe performance of CMR in these patients. ${ }^{33}$ The safety is therefore largely unknown and CMR should be considered to be contraindicated until more information is available.

\section{Insertable loop recorder systems}

Devices such as the Reveal loop recorder are usually inserted subcutaneously in the upper chest area. The combination of the magnetic and RF field may erase or corrupt stored data. ${ }^{34}$ Mechanical forces may cause some local movement of the device. CMR is not contraindicated in these patients provided these considerations are addressed. ${ }^{7}$

\section{Scanning the unstable cardiac patient}

Acutely ill patients are often the group where a definitive diagnosis will make the biggest impact on management and outcome. With proper precautions, these patients can be scanned quickly and safely. Only piped oxygen must be used in the CMR scanner room. Under no circumstances should oxygen cylinders be taken into the scanning room because they may be ferromagnetic. Intravenous pump machines should remain in the control room and connected to the patient with long lines passed through a waveguide. All anaesthetic and trolley equipment should be fully MR compatible. $^{35}$

\section{3-Tesla scanners}

RF heating and mechanical field interaction are related to field strength and are likely to be more problematic at 3T. ${ }^{36}$ Implants with weak ferromagnetic properties at $1.5 \mathrm{~T}$ may demonstrate increased magnetic field interactions at 3T. Much of the current safety assessments cannot therefore simply be extrapolated to the higher field strengths and stricter safety criteria are required. ${ }^{7}$ Certain devices such as heart valve prostheses appear safe at 3T, but wider more definitive evaluation of devices in both long and short bore scanners is still underway and therefore caution should be applied in scanning patients. There is very little clinical CMR being performed at $3 \mathrm{~T}$ in 2004, and no clinical indications at this stage for its use in preference to $1.5 \mathrm{~T}$ where the safety issues are much better documented.

\section{Device evaluation}

In the UK, the main regulatory body is the National Radiation Protection Board (NRPB) which acts as the official advisor to the Department of Health. They provide information on the safety of specific implants and devices. ${ }^{37} \mathrm{~A}$ comprehensive list of implants that have been tested for MR safety or compatibility is readily available. ${ }^{7}$ There are other additional sources of reference available. ${ }^{38-40}$

\section{Future strategies}

Research efforts are underway to improve the safety of CMR studies in patients with pacemakers and ICDs. This includes the use of fibreoptic cardiac pacing leads in place of standard metallic leads, and a low power semiconductor laser to stimulate the heart ${ }^{41}$ The optic cable is very robust and is not affected by the magnetic field or RF heating. Alternatives include decoupling the pacemaker lead to prevent RF heating, as has been achieved for MR interventional guide wires, and improving the immunity of the electronics from interference. It is likely that these new devices will undergo clinical evaluation within the next few years. Work is also underway on artefact-free stents to enable better in-stent visualisation. $^{42}$ 


\section{ACKNOWLEDGEMENTS}

The authors would like to thank Professor David Firmin for review of this editorial.

\section{Authors' affiliations}

S K Prasad, D J Pennell, Cardiovascular Magnetic Resonance Unit, Royal Brompton Hospital, London, UK

\section{REFERENCES}

1 Pohost GM. Is CMR safe? J Cardiovasc Magn Reson 2001 3:ix.

2 Shellock FG. Magnetic resonance safety update 2002: implants and devices J Magn Reson Imaging 2002;16:485-96.

3 Ahmed S, Shellock FG. Magnetic resonance imaging safety: implications for cardiovascular patients. J Cardiovasc Magn Reson 2001;3:171-82.

4 Ho HS. Safety of metallic implants in magnetic resonance imaging. J Magn Reson Imaging 2001;14:472-7.

5 Kanal E, Borgstede JP, Barkovich AJ, et al. American College of Radiology white paper on MR safety. Am J Roentgenol 2002;178:1335-47.

6 Manner I, Alanen A, Komu M, et al. MR imaging in the presence of small circular metallic implants. Assessment of thermal injuries. Acta Radiol 1996;37:551-4.

7 Shellock FG. Reference manual for magnetic resonance safety. WB Saunders, 2002:728-9.

8 Hartnell GG, Spence L, Hughes LA, et al. Safety of MR imaging in patients who have retained metallic materials after cardiac surgery. Am J Roentgenol 1997; 168: 1157-9.

9 Edwards MB, Taylor KM, Shellock FG. Prosthetic heart valves: evaluation of magnetic field interactions, heating, and artifacts at 1.5 T. J Magn Reson Imaging 2000; 12:363-9.

10 Shellock FG. Prosthetic heart valves and annuloplasty rings: assessment of magnetic field interactions, heating, and artifacts at 1.5 Tesla. J Cardiovasc Magn Reson 2001:3:317-24.

11 Strohm O, Kivelitz D, Gross, et al. Safety of implantable coronary stents during $\mathrm{H}-1$ magnetic resonance imaging at 1.0 and 1.5T. J Cardiovasc Magn Reson 2000;1:239-45.

12 Scott NA, Pettigrew RI. Absence of movement of coronary stents afte placement in a magnetic resonance imaging field. Am J Cardiol 1994:73:900-1.

13 Gerber TC, Fasseas P, Lennon RJ, et al. Clinical safety of magnetic resonance imaging early after coronary artery stent placement. J Am Coll Cardiol 2003;42:1295-8

14 Kramer CM, Rogers WJ Jr, Pakstis DL. Absence of adverse outcomes after magnetic resonance imaging early after stent placement for acute myocardia infarction: a preliminary study. J Cardiovasc Magn Reson 2000;2:257-61.

15 Hug J, Nagel E, Bornstedt A, et al. Coronary arterial stents: safety and artifacts during MR imaging. Radiology 2000;216:781-7.

16 Engellau L, Olsrud J, Brockstedt S, et al. MR evaluation ex vivo and in vivo of a covered stent-graft for abdominal aortic aneurysms: ferromagnetism, heating, artifacts, and velocity mapping. J Magn Reson Imaging 2000;12:112-21.

17 Stables RH, Mohiaddin RH, Panting JR, et al. Exclusion of an aneurysmal segment of the thoracic aorta with covered stent. Circulation 2000;101:1888-9.

18 Shellock FG. Reference manual for magnetic resonance safety, 2003 ed. WB Saunders, 2002:260.
19 Razavi R, Hill DL, Keevil SF, et al Cardiac catheterisation guided by MRI in children and adults with congenital heart disease. Lancet 2003;362:1877-82.

20 Achenbach S, Moshage W, et al. Effects of magnetic resonance imaging on cardiac pacemakers and electrodes. Am Heart J 1997;134:467-73.

21 Hayes DL, Holmes DR Jr, Gray JE. Effect of 1.5 Tesla nuclear magnetic resonance imaging scanner on implanted permanent pacemakers. J Am Coll Cardiol 1987; 10:782-6

22 Bhachu DS, Kanal E. Implantable pulse generators (pacemakers) and electrodes: safety in the magnetic resonance imaging scanner environment $J$ Magn Reson Imaging 2000;12:201-4.

23 Avery JE. Loss prevention case of the month. Not my responsibility! J Tenn Med Assoc, 1988:81:523.

24 Schiebler M, Kaut-Watson C, Williams DL. Both sedated and critically ill require monitoring during MRI. Winter MR 1994:41-5.

25 US Food and Drug Administration, Centre for Devices and Radiological Health. MR product reporting program and medical device report program (microfiche). MDR \# 175218. \#351516, \#125938.

26 Luechinger R, Duru F, Zeijlemaker VA, et al. Pacemaker reed switch behavior in $0.5,1.5$, and 3. 0 Tesla magnetic resonance imaging units: are reed switches always closed in strong magnetic fields, PACE 2002;25:1419-23.

27 Alagona P, Toole JC, Maniscalco BS, et al. Nuclear magnetic resonance imaging in a patient with a DDD pacemaker. PACE 1989;12:619.

28 Sommer T, Vahlhaus C, Lauck G, et al. MR imaging and cardiac pacemakers: in vitro evaluation and in vivo studies in 51 patients at $0.5 \mathrm{~T}$. Radiology 2000;215:869-79.

29 Gimbel JR, Johnson D, Levine PA, et al. Safe performance of magnetic resonance imaging on five patients with permanent cardiac pacemakers. PACE 1996:19:913.

30 Pennell DJ. Cardiac magnetic resonance with a pacemaker in-situ: can it be done? J Cardiovasc Magn Reson 1999;1:72 [abstract]

31 Martin ET, Coman JA, Shellock FG, et al. Magnetic resonance imaging and cardiac pacemaker safety at 1.5-Tesla. J Am Coll Cardiol 2004:43:1315-24.

32 Shellock FG, Tkach JA, Ruggieri PM, et al. Cardiac pacemakers, ICDs, and loop recorder: evaluation of translational attraction using conventional ("longbore" $^{\prime \prime}$ ) and "short-bore" 1.5- and 3.0-Tesla MR systems. J Cardiovasc Magn Reson 2003:5:387-97.

33 Gimbel JR, Trohman RL, Lindsay WC, et al. Strategies for the safe performance of magnetic resonance imaging in selected ICD patients. PACE 2002;24:618 [abstract].

34 Gimbel JR, Wilkoff BL. Artefact mimicking tachycardia during magnetic resonance imaging in a patient with an implantable loop recorder. Heart 2003;89:e10

35 Laurence AS. Magnetic resonance compatible equipment: apply common sense. Anaesthesia 2003;58:615.

36 Takahashi $M$, Uematsu $H$, Hatabu $H$. MR imaging at high magnetic fields. Eur J Radiol 2003;46:45-52.

37 National Radiation Protection Board. www.nrpb.org.uk.

38 www.mrisafety.com.

39 www.radiology.upmc.edu/MRsafety.

40 www.IMSER.org.

41 Greatbatch W, Miller V, Shellock FG. Magnetic resonance safety testing of a newly-developed fiber-optic cardiac pacing lead. J Magn Reson Imaging 2002; 16:97-103

42 Buecker A, Spuentrup E, Ruebben A, et al. Artifact-free in-stent lumen visualization by standard magnetic resonance angiography using a new metallic magnetic resonance imaging stent. Circulation 2002; 105:1772-5.

\section{Call for papers}

10th European Forum on Quality Improvement in Health Care

13-15 April 2005, ExCel, Docklands, London

For further information on how to submit your paper please go to:

http://www.quality.bmipg.com 